\title{
Probiotics and virulent human rotavirus modulate the transplanted human gut microbiota in gnotobiotic pigs
}

\author{
Husen Zhang ${ }^{1 \dagger}$, Haifeng Wang ${ }^{2,5+}$, Megan Shepherd ${ }^{3}$, Ke Wen ${ }^{2}$, Guohua Li ${ }^{2}$, Xingdong Yang ${ }^{2}$, Jacob Kocher ${ }^{2}$, \\ Ernawati Giri-Rachman ${ }^{2}$, Allan Dickerman ${ }^{4}$, Robert Settlage ${ }^{4}$ and Lijuan Yuan ${ }^{2 *}$
}

\begin{abstract}
We generated a neonatal pig model with human infant gut microbiota (HGM) to study the effect of a probiotic on the composition of the transplanted microbiota following rotavirus vaccination and challenge. All the HGM-transplanted pigs received two doses of an oral attenuated rotavirus vaccine. The gut microbiota of vaccinated pigs were investigated for effects of Lactobacillus rhamnosus GG (LGG) supplement and homotypic virulent human rotavirus (HRV) challenge. High-throughput sequencing of V4 region of $16 \mathrm{~S}$ rRNA genes demonstrated that HGM-transplanted pigs carried microbiota similar to that of the C-section delivered baby. Firmicutes and Proteobacteria represented over $98 \%$ of total bacteria in the human donor and the recipient pigs. HRV challenge caused a phylum-level shift from Firmicutes to Proteobacteria. LGG supplement prevented the changes in microbial communities caused by HRV challenge. In particular, members of Enterococcus in LGG-supplemented pigs were kept at the baseline level, while they were enriched in HRV challenged pigs. Taken together, our results suggested that HGM pigs are valuable for testing the microbiota's response to probiotic interventions for treating infantile HRV infection.
\end{abstract}

Keywords: Microbiota, Gnotobiotic pigs, Rotavirus, Vaccine, Lactobacillus, Probiotics

\section{Introduction}

Humanized microbiota models, such as germ-free animals transplanted with human feces, are valuable for studying microbiota composition change due to external factors by minimizing confounding variables $[1,2]$. Gnotobiotic (Gn) pigs provide an excellent model for isolating microbiota as an environmental factor in disease models [3] because pigs and human share high genome homology (98\%), similar intestinal anatomy, physiology, immune systems, nutritional requirements, and food transit times [4-6]. Both pigs and human are also susceptible to human rotavirus Wa strain (genotype G1P [7]) infection and disease [8].

Human rotavirus (HRV) infection is the leading cause of gastroenteritis in infants and children, especially in

\footnotetext{
* Correspondence: lyuan@vt.edu

${ }^{\dagger}$ Equal contributors

${ }^{2}$ Department of Biomedical Sciences and Pathobiology, Virginia-Maryland Regional College of Veterinary Medicine, Virginia Polytechnic Institute and State University, Blacksburg, VA 24061, USA

Full list of author information is available at the end of the article
}

developing countries [7]. The burden is exacerbated for infants and young children in the developing world, because many of them have a weak immune response to oral rotavirus vaccines and the protective efficacies of the vaccines are lower compared to that in the developed world [9]. The gut microbiota's response to rotavirus infection has not been systematically investigated; despite that, microbiota disruption (intestinal dysbiosis) may be a risk factor for long-term adverse effects. Rotavirus infection in humans was found to be associated with an increase in Bacteroides fragilis and decreased $B$. vulgatus and B. stercoris in a clone-library study [10]. However, a comprehensive analysis is lacking for the effect of therapeutic interventions such as vaccination and probiotics on rotavirus-infected gut microbiota.

Orally administered probiotic bacterium Lactobacillus rhamnosus GG (LGG) has been tested in numerous clinical trials to prevent or shorten rotavirus-induced diarrhea $[11,12]$. Supplementation with LGG for 4 weeks after acute rotavirus infection reduced intestinal permeability in 
children with rotavirus diarrhea, reduced the number of subsequent diarrheal episodes and increased IgG antibody response [13]. Lactobacilli have also been implicated in lipid metabolism due to their bile salt hydrolase activities [14]. We hypothesized that the beneficial effects of LGG against rotavirus-related diarrhea may be a result of modulating the intestinal microbiota towards a healthier profile.

We aimed to determine how rotavirus infection affects the human gut microbiota inoculated in Gn pigs and whether probiotic LGG can prevent the disruption of the microbiota. We transplanted human infant fecal microbiota to newborn Gn pigs. The pigs were or were not treated with a daily dose of LGG for 2 weeks, vaccinated with an oral attenuated HRV vaccine, and subsequently challenged or not challenged with virulent HRV. We investigated: (a) how efficiently different bacterial species in the human gut microbiota (HGM) colonize neonatal germ-free pigs, (b) the associative changes in the microbiota in response to HRV challenge, and (c) whether LGG, HRV and interactions between LGG and HRV have effects on the gut microbial community structure.

\section{Materials and methods}

\section{Ethics statement}

All animal experiments were performed in strict accordance with federal and university guidelines. Specifically, we adhered to the recommendations in the Guide for the Care and Use of Laboratory Animals of the National Institutes of Health and the American Veterinary Medical Association Guidelines on Euthanasia. The animal protocol was approved by the Institutional Animal Care and Use Committee at Virginia Tech (Protocol\# 10-168CVM and 13-187-CVM). Ethical Committee approval was received from Virginia Tech Institutional Review Board for the newborn human stool sample collection (IRB number 11-1049).

\section{Transplantation of HGM into Gn pigs}

Gn pigs were derived by hysterectomy from near-term sows (Landrace and Large White crossbred) and maintained in germ-free isolator units $[8,15]$. Pigs of the same treatment groups were housed in individual 4-place isolators. The pigs were fed ultra-high-temperature sterilized milk (Hershey) throughout the experiment.

Multiple stool samples from a cesarean-section delivered, exclusively breast-fed healthy infant at 17-23 days of age were collected and made into an inoculum pool to generate HGM pigs. Briefly, daily collected fresh stool was diluted 20-fold in sterile pre-reduced PBS ( $\mathrm{pH} 7.2$ ) and glycerol ( $15 \%$ by volume) and stored at $-80^{\circ} \mathrm{C}$ under an atmosphere of nitrogen. A week-long multiple stool samples were pooled and homogenized for use in the entire course of the experiment. Prior to inoculation, the human stool was screened for pathogens as previously described [16]. The screening showed no hemolytic activity. Next-generation sequencing at Viral Diagnostics and Discovery Center at UCSF showed no known viruses in the sample. Pigs were orally inoculated with the human stool inoculum ( $1 \mathrm{ml}$ of $5 \%$ stool suspension in PBS) once daily starting at 12 hours after birth for three days to establish the HGM in Gn pigs. The timing of HGM inoculation is to mimic the natural microbial colonization of the newborn's gut (within hours after birth).

\section{Inoculation of Gn pigs with attenuated HRV vaccine, virulent HRV and probiotics (LGG)}

The cell-culture adapted attenuated HRV Wa strain (G1P1A [7]) was used as the vaccine at a dose of $5 \times 10^{7}$ fluorescent focus forming units (FFU) [8]. The virulent HRV Wa strain was passaged through Gn pigs and the pooled intestinal contents were used for challenge of $\mathrm{Gn}$ pigs at a dose of $\sim 10^{5}$ FFU [17]. The virus titer was determined by using cell culture immunofluorescence (CCIF) assay and was expressed as $\mathrm{FFU} / \mathrm{ml}$ as described previously [18]. Probiotic LGG (ATCC\# 53103) was propagated in Lactobacilli MRS broth (Weber, Hamilton, NJ, USA). LGG inoculums were prepared and titrated as previously described [19].

All the HGM pigs received two dose of the oral attenuated HRV vaccine at 5 and 15 days of age. The purpose of the vaccination is to study the effects of LGG in enhancing the immunogenicity of rotavirus vaccines in the HGM pigs, which was the objective of another concurrent study [16]. The HGM-transplanted and vaccinated pigs were divided into four groups: (a) no LGG feeding, no virulent HRV challenge (-LGG-HRV, $\mathrm{n}=4$ ), (b) no LGG feeding, with HRV challenge (-LGG + HRV, $\mathrm{n}=4$ ), (c) with LGG feeding, no HRV challenge (+LGG-HRV, $\mathrm{n}$ =4), and (d) with both LGG feeding and HRV challenge $(+\mathrm{LGG}+\mathrm{HRV}, \mathrm{n}=3$, one pig was euthanized prior to the scheduled time due to health problems). Daily LGG feeding started at 3 days of age for 14 days (3-16 days of age) with 10-fold incremental LGG dose increase every day (from $10^{3}$ to $10^{9} \mathrm{CFU} /$ dose) as previously described [19]. LGG was administrated in $3 \mathrm{ml}$ of $0.1 \%$ peptone water. Non-LGG fed pigs were given $3 \mathrm{ml}$ of $0.1 \%$ peptone water but no LGG. Pigs in the + HRV groups were challenged with the virulent $\mathrm{HRV}$ at post-attenuated HRV inoculation day (PID) 28. Pigs were euthanized at PID 28 before challenge (-LGG-HRV and + LGG-HRV groups) or at post-challenged day (PCD) 7 (-LGG + HRV and + LGG + HRV groups). The pig body weight at euthanasia did not differ among different treatment groups. Colonic contents and serum samples were collected at euthanasia as previously described [16]. After virulent HRV challenge, rotavirus diarrhea and fecal virus shedding were monitored from PCD 1 to 7 (14). 


\section{Microbial community analysis}

Colonic contents from the pig large intestine were collected at euthanasia and stored at $-80^{\circ} \mathrm{C}$. DNA from human stools and pig intestinal contents was extracted with the QIAamp stool mini kit following manufacture's instructions. The 16S rRNA gene amplicons were generated by PCR with $515 \mathrm{~F}$ and barcoded 806R primers [20]. Purified amplicons were sequenced with Illumina MiSeq ${ }^{\mathrm{mm}}$.

Sequencing reads were processed with Quantitative Insights Into Microbial Ecology (QIIME) [21]. High quality reads with Phred quality score $\geq 20$ (corresponding to an sequencing error rate $\leq 0.01$ ) were clustered into operational taxonomic units (OTUs) with the program UCLUST [22]. Chimeric sequences were identified with CHIMERASLAYER [23] and removed from further analysis. Bacterial taxonomy was assigned by using a naïve Bayes classifier [24] against reference databases and bacterial taxonomy maps at Greengenes [25]. A phylogenetic tree was constructed [26] from PyNAST-aligned sequences representing each OTU. Principle coordinate analysis on stool samples was based on UniFrac distances [27]. Distance-based redundancy analysis for effect of HRV on community structures was performed with the VEGAN package [28]. Shannon and Simpson diversity indices and a rank abundance curve were both generated with QIIME.

The nucleotide sequences have been deposited to MGRAST [29] with the accession number 4547774.3. Comparison of data with respect to LGG and HRV treatment was done with unpaired t-tests, Mann-Whitney test without assuming normal distributions, One-Way or Two-Way ANOVA.

\section{Results}

A total of 5,616,353 non-chimeric high quality sequences from feces of the human donor and the recipient pigs were analyzed with QIIME. We analyzed the sequences at the operational taxonomic unit (OTU) level [30]. The recipient pigs carried microbiota that are similar to the human donor's microbiota (Figure 1A), despite that all pigs had received the attenuated HRV vaccine. Two

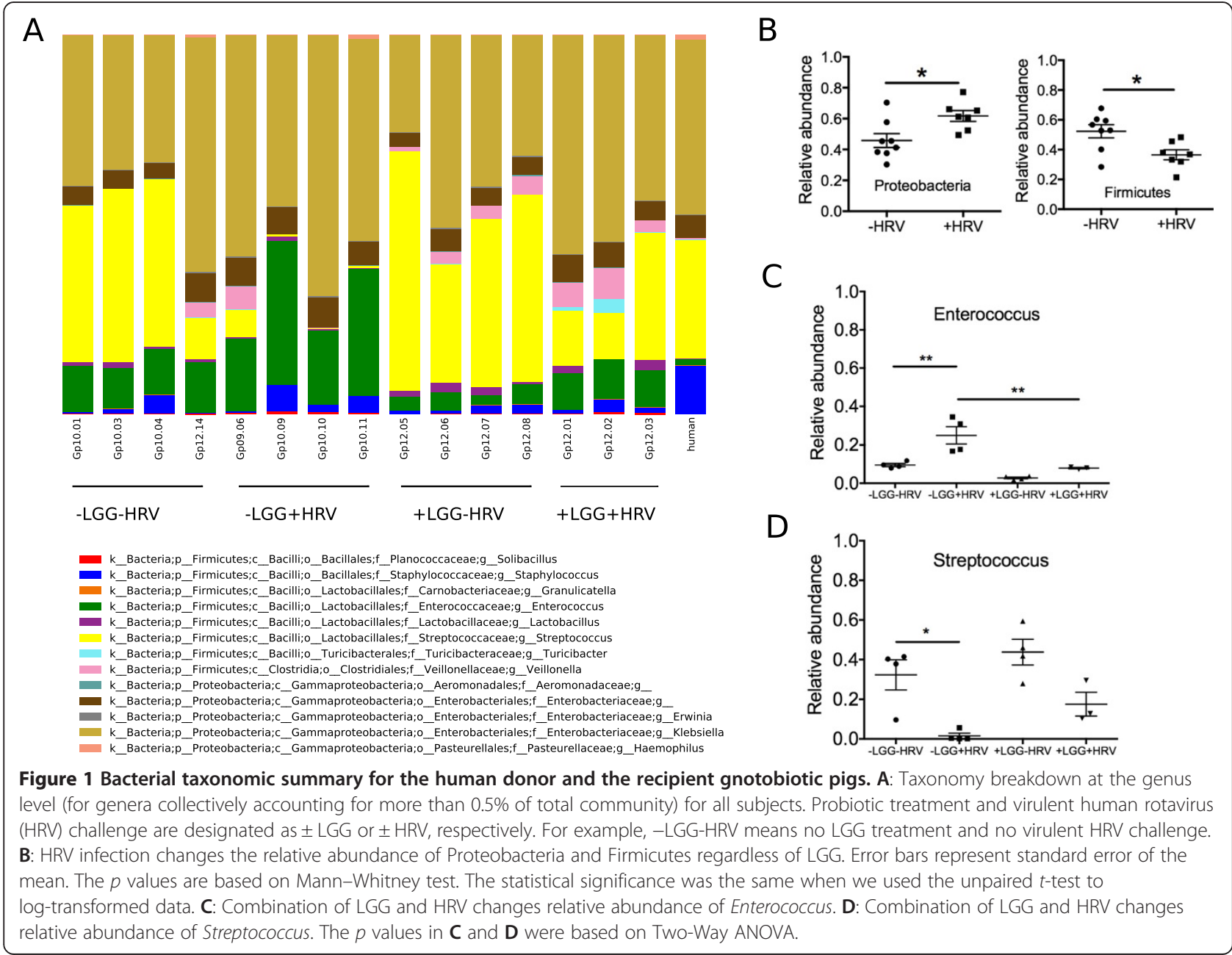


bacterial phyla, Firmicutes and Proteobacteria, representing over $98 \%$ of total bacterial sequences in each subject, dominated microbiota of both the human donor (delivered by $\mathrm{C}$-section) and the recipient pigs. The most abundant genera within Firmicutes were Streptococcus, Enterococcus, Veillonella, and Staphylococcus (Figure 1A). The rank abundance curve showed that the gut microbiota of human and pigs had a long tail of rare OTUs (Additional file 1: Figure S1). For example, around 900 OTUs (species rank from 100 to 1000) each accounted only $0.01 \%\left(10^{-4}\right)$ to well below $0.001 \%\left(10^{-5}\right)$ of total bacteria. The human gut microbiota represented by the dashed line appeared to be above the gut microbiota of recipient pigs, suggesting a slightly higher evenness of human microbiota compared with pigs.

We observed a shift in the microbiota composition at phylum, genus, and OTU levels. At the phylum level, the relative abundance of Proteobacteria and Firmicutes in transplanted pigs was affected by HRV challenge (Figure 1B). HRV-challenged pigs had 16\% less Firmicutes, and accordingly $16 \%$ more Proteobacteria, than nonchallenged pigs. The significance of the shift was confirmed by Mann-Whitney test $(P<0.05$ for both phyla). The phyla Proteobacteria harbor many aerobes and facultative anaerobes, and could serve important roles in removing oxygen diffused from the gut epithelium [31]. We also found that HRV challenge was a factor in changes in overall community structures at the OTU level (Additional file 1: Figure S2), based on results from distance-based redundancy analysis (db-RDA). At the genus level, two abundant genera, Enterococcus and Streptococcus, were affected by LGG feeding and HRV challenge. In the absence of LGG feeding, HRVchallenged pigs had significantly elevated Enterococcus over non-challenged pigs $(P<0.01$, Figure $1 C)$. This effect was absent for pigs fed with LGG, indicating that LGG prevented HRV's effect on Enterococcus. In HRVchallenged pigs, LGG-treated pigs again had significantly reduced Enterococcus $(P<0.01$, Figure $1 C)$. HRV decreased Streptococcus in pigs without LGG feeding $(P<$ 0.05 , Figure 1D), but the effect disappeared in LGG-fed pigs, suggesting again that LGG prevented microbiota perturbation by rotavirus infection. The combination of LGG and HRV also affected less abundant genera including Veillonella and Aeromonas (data not shown). Similar to our findings, an increase in Proteobacteria was reported in norovirus-infected humans [32].

We were interested in whether HRV and/or LGG changed overall community richness and evenness. We found that HRV challenge had no significant effect on Shannon or Simpson diversity indices. LGG-treated microbiota appeared to be slightly more diverse than untreated microbiota, but the effect was not significant (Figure 2).

To analyze LGG's effect on microbial community structures, we performed principal coordinate analysis (PCoA) on weighted UniFrac distances. The results showed that LGG-treated pig microbiota was distinct from those receiving no LGG (Figure 3A), supported by a permutational multivariate analysis (PERMANOVA) with a $p$ value of 0.005 at 999 permutations [33]. The human microbiota appeared to cluster closer with -LGG pigs. Figure 3B showed that the extent to which HRV changed microbiota depended on LGG. The HRV-caused microbiota change, measured by UniFrac distances between + HRV and HRV pigs, was smaller for LGG treated pigs than for noLGG treated pigs $(p<0.001$, Figure $3 \mathrm{~B})$, suggesting an interaction between LGG and HRV on the microbiota structure. Overall, LGG treatment could resist the change of microbial community structures caused by HRV challenge.
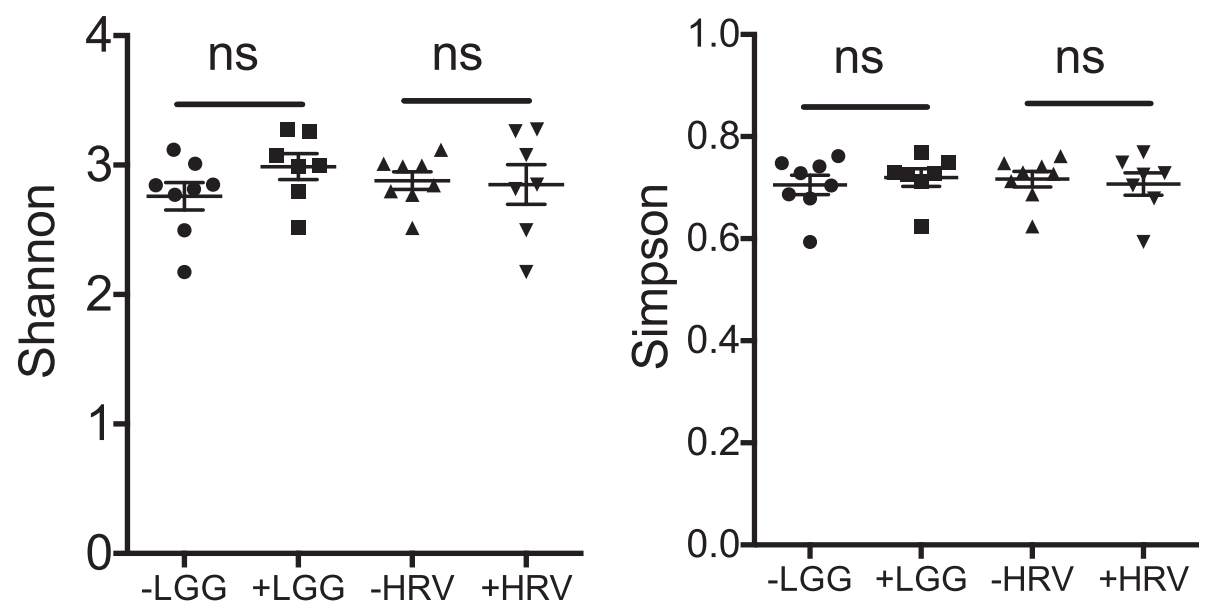

Figure 2 Shannon $(\mathrm{H})$ and Simpson (E) diversity indices of pig microbiota with respect to LGG and HRV treatment. Values for the human donor microbiota are: $\mathrm{H}=3.017$ and $\mathrm{E}=0.757$. ns: not significant based on unpaired $t$-tests. 


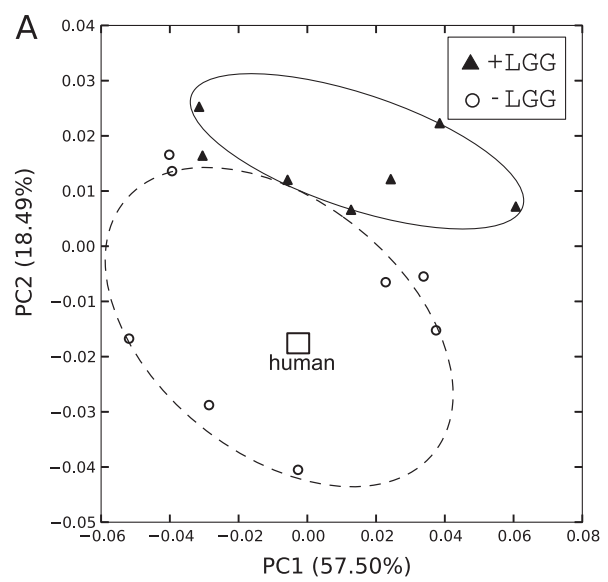

B

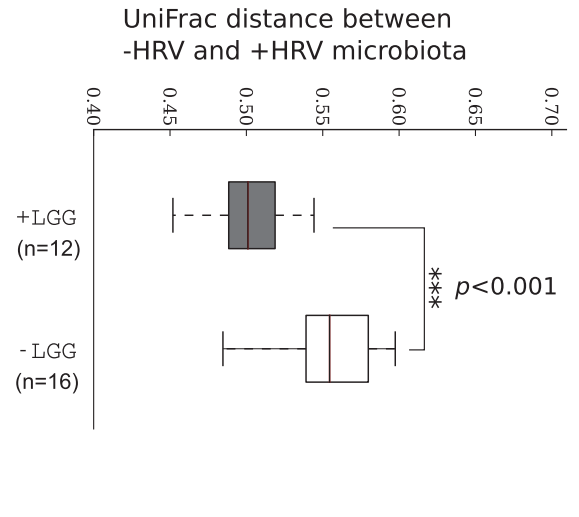

Figure 3 Phylogenetic dissimilarities among transplanted pigs and the effect of LGG treatment. A: A principal coordinate (PCoA) analysis of weighted UniFrac distances among all pigs. Only the first two axes (PC1 and PC2) that explain largest variations among samples are plotted. Open circles are pigs receiving no LGG, and filled triangles are pigs treated with LGG. The open square indicates the human microbiota. Significant grouping by LGG was tested by PERMANOVA described in the text. B: The UniFrac distances between HRV-challenged pig microbiota versus non-challenged pig microbiota. These distances were divided into two groups according to whether LGG was added. The test of significance was performed using the Mann-Whitney test.

\section{Discussion}

In this study, we demonstrated that human gut microbiota could be transplanted to and colonize gnotobiotic pigs. The resulting "humanized pigs" share the majority of human donor microbiota, albeit the human donor microbiota appeared to be more even than the colonized pig gut microbiota. Virulent rotavirus challenge changed Enterococcus and Streptococcus abundance in the humanized pig microbiota. Adding probiotic LGG prevented these changes.

The human infant microbiota used in our study was a composite sample from a week-long daily collection of feces. Our intention was to reduce the temporal variations and dynamics known for human microbiota of this age $[34,35]$. We acknowledge that effect of inter-personal microbiota variations on colonization of $\mathrm{Gn}$ pigs would need to be evaluated through the use of fecal samples from a higher number of infants. Moreover, the inter-pig variations in HGM colonization could be minimized through the use of highly in-bred pigs. Overcoming these limitations will allow us to quantify how stable the microbiota of recipient pigs are.

We chose a C-section delivered HGM donor in this study because of the increasing popularity of this delivery mode. The dominance of Firmicutes and Proteobacteria while lacking Actinobacteria and Bacteroidetes is typical for $\mathrm{C}$-section delivered babies and different from vaginallydelivered babies [36,37]. In particular, Bifidobacteria, a prominent group in the phylum Actinobacteria, were absent in microbiota from C-section delivered infants, but was found abundant in vaginally-delivered infants [36]. In another high-throughput sequencing study, the mode of delivery was found to be the main determinant of newborn's microbiota [37]. In that study, C-section delivered babies lacked mothers' vaginal species within Actinobacteria; Bacteroidetes was found to be mainly in the vaginal delivered babies. The same study also reported that Staphylococcus species appeared in C-section delivered babies, which agreed with our results (Figure 1A, Staphylococcus in dark blue color).

Previous studies have demonstrated that the oral HRV vaccine does not alter the gut microbiota in older children [38]. This provided rationale for us not to include HMG pigs without an HRV vaccine. All the Gn piglets in our study were vaccinated with attenuated HRV, yet the microbiota in the vaccinated pigs was still significantly altered by virulent HRV (Figure 1B and Additional file 1: Figure S2). We speculate that the extent of microbiota alterations caused by virulent HRV would have been larger if the Gn pigs were not vaccinated. This hypothesis warrants further investigation, as most children in developing countries are not vaccinated against HRV. We found LGG could prevent certain changes in microbiota induced by HRV, suggesting potential interacting effects of LGG and HRV on microbiota, although the exact nature of such interactions is unclear.

Szajewska et al. [11] demonstrates that LGG improves HRV-induced diarrhea. That study used subjects 1 month to 18 years old but did not specify the delivery type. It is possible that LGG may not have the same protective effects on C-section delivered infants which contain a different gut microbiota compared with vaginal delivered infants. Although all the pigs studied received the oral attenuated $H R V$ vaccine, the protection against virulent $H R V$ induced diarrhea was only partial [16]. There were no significant differences in protection rate against diarrhea or virus 
shedding, the severity of diarrhea, or the titer of virus shedding (data not shown); therefore we could not evaluate quantitatively whether the changes in the microbiome correlate with protection against diarrhea or virus shedding. Fifty percent (2/4) of the pigs in the -LGG + HRV group were protected from infection upon virulent HRV challenge. There is no apparent difference in the abundant taxa between protected (Gp09.06 and Gp10.09) versus unprotected (Gp10.10 and Gp10.11) pigs (Figure 1A, group "-LGG + HRV"). However, the low abundance bacterial taxa (Additional file 1: Figure S3), which accounted for less than $0.5 \%$ of the microbiota, showed that one of the two protected pigs (Gp09.06) harbored unique bacteria such as Ruminococcaceae. The other protected pig (Gp10.09) shared similar low-abundance taxa with unprotected pigs. Further studies with more animals would be needed to identify rare taxa potentially associated with viral protection.

Several relevant issues that were not resolved in the present study will be addressed in future studies. These include (a) the effect of vaccination on the gut microbiota. (b) Microbiota in human infants varies among individuals and changes during the first few months of life, especially at weaning. Our study used HGM from only one C-section delivered newborn; a HGM mixture from multiple older children matching the age of rotavirus vaccination (e.g., 2 to 6 months) will be a better model for vaccine evaluation. Additional data from both Csection-derived and conventional birth-derived infants would be highly desirable to further evaluation the HGM pig model. (c) The LGG feeding in this study did not significantly improve the protection conferred by the rotavirus vaccine. Changes in the microbiome due to LGG feeding were not associated with a change in the protective efficacy of the vaccine. Adjustment of the dose and dosing regimen of LGG may be needed in order for LGG to exert its adjuvant effect on the rotavirus vaccine. The microbiome structure and composition that may favor stronger immunogenicity and protective efficacy of rotavirus vaccines require further studies to identify.

In conclusion, the HGM-transplanted gnotobiotic pig presents a useful model for testing interventions using probiotics and vaccines to prevent or treat infantile diarrhea and improve enteric health and immunity. Future study using a mixture of feces from multiple older children that match the age of rotavirus vaccination will improve this model for vaccine evaluation.

\section{Additional file}

Additional file 1: Figure S1. Relative abundance of OTUs plotted against OTU rank. From left to right, high-ranking OTU with high abundance towards low-ranking OTU with low abundance.
Figure S2. Distance-based redundancy analysis (db-RDA) of pig microbiota in response to HRV challenge. CAP1 and MDS1 are constrained and unconstrained axes, respectively. HRV-challenged pigs are indicated by ovals, and LGG supplemented pigs are indicated by underlines. The arrow indicates source of variation explained by HRV. Figure S3. Rare taxa (OTUs $<0.5 \%$ of total bacteria) in the -LGG+HRV group. Gp09.06 and Gp 10.09 were protected from infection by attenuated HRV vaccines. Gp 10.10 and Gp10.11 were unprotected.

\section{Competing interests}

The authors declare that they have no competing interests.

\section{Authors' contributions}

LY designed the study. HW, KW, GL, XY, JK, EGR carried out the experiments, $H Z, A D, R S$ performed data analysis. HZ, MS, LY wrote the manuscript. All authors read and approved the final manuscript.

\section{Acknowledgments}

We thank Dr. Kevin Pelzer and Dr. Sherrie Clark-Deener for veterinarian service, Pete Jobst, Andrea Pulliam, Kimberly Allen, Shannon Viers, and Mariah Weiss for animal care. This work was supported by a grant to LY (R01AT004789) from the National Center of Complementary and Alternative Medicine, National Institutes of Health, Bethesda, MD.

\section{Author details}

${ }^{1}$ Department of Civil and Environmental Engineering, Virginia Polytechnic Institute and State University, Blacksburg, VA 24061, USA. ²Department of Biomedical Sciences and Pathobiology, Virginia-Maryland Regional College of Veterinary Medicine, Virginia Polytechnic Institute and State University, Blacksburg, VA 24061, USA. ${ }^{3}$ Department of Large Animal Clinical Sciences, Virginia-Maryland Regional College of Veterinary Medicine, Virginia Polytechnic Institute and State University, Blacksburg, VA 24061, USA. ${ }^{4}$ Virginia Bioinformatics Institute, Blacksburg, VA 24061, USA. ${ }^{5}$ College of Animal Science \& Technology, Zhejiang A \& F University, Zhejiang Province, China.

Received: 1 August 2014 Accepted: 1 September 2014 Published: 9 September 2014

\section{References}

1. Turnbaugh PJ, Ridaura VK, Faith JJ, Rey FE, Knight R, Gordon Jl: The effect of diet on the human gut microbiome: a metagenomic analysis in humanized gnotobiotic mice. Sci Transl Med 2009, 1:6ra14-6ra14.

2. Fei $N$, Zhao $L$ : An opportunistic pathogen isolated from the gut of an obese human causes obesity in germfree mice. ISME J 2013, 7:880-884.

3. Zhang Q, Widmer G, Tzipori S: A pig model of the human gastrointestinal tract. Gut Microbes 2013, 4:193-200.

4. Fritz JV, Desai MS, Shah P, Schneider JG, Wilmes P: From meta-omics to causality: experimental models for human microbiome research. Microbiome 2013, 1:14

5. Dawson HD, Loveland JE, Pascal G, Gilbert JG, Uenishi H, Mann KM, Sang Y, Zhang J, Carvalho-Silva D, Hunt T, Hardy M, Hu Z, Zhao S-H, Anselmo A, Shinkai H, Chen C, Badaoui B, Berman D, Amid C, Kay M, Lloyd D, Snow C, Morozumi T, Cheng RP-Y, Bystrom M, Kapetanovic R, Schwartz JC, Kataria R, Astley M, Fritz E, et al: Structural and functional annotation of the porcine immunome. BMC Genomics 2013, 14:332.

6. Meurens F, Summerfield A, Nauwynck H, Saif L, Gerdts V: The pig: a model for human infectious diseases. Trends Microbiol 2012, 20:50-57.

7. Tate JE, Burton AH, Boschi-Pinto C, Steele AD, Duque J, Parashar UD: 2008 estimate of worldwide rotavirus-associated mortality in children younger than 5 years before the introduction of universal rotavirus vaccination programmes: a systematic review and meta-analysis. Lancet Infect Dis 2012, 12:136-141.

8. Yuan L, Ward LA, Rosen BI, To TL, Saif L: Systematic and intestinal antibody-secreting cell responses and correlates of protective immunity to human rotavirus in a gnotobiotic pig model of disease. J Virol 1996, 70:3075-3083

9. Madhi SA, Cunliffe NA, Steele D, Witte D, Kirsten M, Louw C, Ngwira B, Victor JC, Gillard PH, Cheuvart BB, Han HH, Neuzil KM: Effect of human 
rotavirus vaccine on severe diarrhea in African infants. N Engl J Med 2010, 362:289-298.

10. Zhang M, Zhang M, Zhang C, Du H, Wei G, Pang X, Zhou H, Liu B, Zhao L: Pattern extraction of structural responses of gut microbiota to rotavirus infection via multivariate statistical analysis of clone library data. FEMS Microbiol Ecol 2009, 70:177-185.

11. Szajewska $H$, Wanke M, Patro B: Meta-analysis: the effects of Lactobacillus rhamnosus GG supplementation for the prevention of healthcareassociated diarrhoea in children. Aliment Pharmacol Ther 2011, 34:1079-1087.

12. Isolauri $E$, Juntunen $M$, Rautanen $T$, Sillanaukee $P$, Koivula $T$ : $A$ human Lactobacillus strain (Lactobacillus casei sp strain GG) promotes recovery from acute diarrhea in children. Pediatrics 1991, 88:90-97.

13. Sindhu KNC, Sowmyanarayanan TV, Paul A, Babji S, Ajjampur SSR, Priyadarshini S, Sarkar R, Balasubramanian KA, Wanke CA, Ward HD, Kang G: Immune response and intestinal permeability in children with acute gastroenteritis treated with Lactobacillus rhamnosus GG (LGG): a randomized, double-blinded, placebo-controlled trial. Clin Infect Dis 2014, 58(8):1107-1115. doi: 10.1093/cid/ciu065.

14. Wang Z, Zeng $X$, Mo $Y$, Smith $K$, Guo $Y$, Lin J: Identification and characterization of a bile salt hydrolase from lactobacillus salivarius for development of novel alternatives to antibiotic growth promoters. Appl Environ Microbiol 2012, 78:8795-8802.

15. Meyer RC, Bohl EH, Kohler EM: Procurement and maintenance of germfree seine for microbiological investigations. Appl Microbiol 1964, 12:295-300.

16. Wen K, Tin C, Wang H, Yang X, Li G, Giri-Rachman E, Kocher J, Bui T, Clark-Deener S, Yuan L: Probiotic lactobacillus rhamnosus GG enhanced Th1 cellular immunity but did not affect antibody responses in a human gut microbiota transplanted neonatal gnotobiotic pig model. PLOS One 2014, 9:e94504.

17. Yuan L, Wen K, Azevedo MSP, Gonzalez AM, Zhang W, Saif LJ: Virus-specific intestinal IFN-gamma producing $T$ cell responses induced by human rotavirus infection and vaccines are correlated with protection against rotavirus diarrhea in gnotobiotic pigs. Vaccine 2008, 26:3322-3331.

18. Liu F, Li G, Wen K, Bui T, Cao D, Zhang Y, Yuan L: Porcine small intestinal epithelial cell line (IPEC-J2) of rotavirus infection as a new model for the study of innate immune responses to rotaviruses and probiotics. Viral Immunol 2010, 23:135-149.

19. Liu F, Wen K, Li G, Yang X, Kocher J, Bui T, Jones D, Pelzer K, Clark-Deener S, Yuan L: Dual functions of lactobacillus acidophilus NCFM as protection against rotavirus diarrhea. J Pediatr Gastroenterol Nutr 2014, 58:171-178.

20. Caporaso JG, Lauber CL, Walters WA, Berg-Lyons D, Huntley J, Fierer N, Owens SM, Betley J, Fraser L, Bauer M, Gormley N, Gilbert JA, Smith G, Knight R: Ultra-high-throughput microbial community analysis on the Illumina HiSeq and MiSeq platforms. ISME J 2012, 6:1621-1624.

21. Caporaso JG, Kuczynski J, Stombaugh J, Bittinger K, Bushman FD, Costello EK, Fierer N, Pena AG, Goodrich JK, Gordon Jl, Huttley GA, Kelley ST, Knights D, Koenig JE, Ley RE, Lozupone CA, McDonald D, Muegge BD, Pirrung M, Reeder J, Sevinsky JR, Turnbaugh PJ, Walters WA, Widmann J, Yatsunenko T, Zaneveld J, Knight R: QIIME allows analysis of high-throughput community sequencing data. Nat Methods 2010, 7:335-336.

22. Edgar RC: Search and clustering orders of magnitude faster than BLAST. Bioinformatics 2010, 26:2460-2461.

23. Haas BJ, Gevers D, Earl AM, Feldgarden M, Ward DV, Giannoukos G, Ciulla D, Tabbaa D, Highlander SK, Sodergren E, Methé B, DeSantis TZ, Petrosino JF, Knight R, Birren BW: Chimeric 16S rRNA sequence formation and detection in Sanger and 454-pyrosequenced PCR amplicons. Genome Res 2011, 21:494-504.

24. Wang Q, Garrity GM, Tiedje JM, Cole JR: Naive Bayesian classifier for rapid assignment of rRNA sequences into the new bacterial taxonomy. Appl Env Microbiol 2007, 73:5261-5267.

25. McDonald D, Price MN, Goodrich J, Nawrocki EP, DeSantis TZ, Probst A, Andersen GL, Knight R, Hugenholtz P: An improved Greengenes taxonomy with explicit ranks for ecological and evolutionary analyses of bacteria and archaea. ISME J 2012, 6:610-618.

26. Price MN, Dehal PS, Arkin AP: FastTree 2-approximately maximumlikelihood trees for large alignments. PLoS One 2010, 5:e9490.

27. Lozupone C, Knight R: UniFrac: a new phylogenetic method for comparing microbial communities. Appl Environ Microbiol 2005, 71:8228-8235.
28. Jari O, Guillaume Blanchet F, Roeland K, Pierre L, Minchin PR, O'Hara RB, Simpson GL, Peter Solymos M, Henry H: Stevens and Helene Wagner. Vegan: Community Ecology Package; 2013.

29. Meyer F, Paarmann D, D'Souza M, Olson R, Glass EM, Kubal M, Paczian T, Rodriguez A, Stevens R, Wilke A, Wilkening J, Edwards RA: The metagenomics RAST server - a public resource for the automatic phylogenetic and functional analysis of metagenomes. BMC Bioinformatics 2008, 9:386.

30. Schloss PD, Handelsman J: Introducing DOTUR, a computer program for defining operational taxonomic units and estimating species richness. Appl Env Microbiol 2005, 71:1501-1506.

31. Pedron T, Mulet C, Dauga C, Frangeul L, Chervaux C, Grompone G, Sansonetti PJ: A crypt-specific core microbiota resides in the mouse colon. mBio 2012, 3:e00116-12-e00116-12.

32. Nelson AM, Walk ST, Taube S, Taniuchi M, Houpt ER, Wobus CE, Young VB: Disruption of the human gut microbiota following norovirus infection. Plos One 2012, 7:e48224

33. Anderson MJ: A new method for non-parametric multivariate analysis of variance. Austral Ecol 2001, 26:32-46.

34. Palmer C, Bik EM, DiGiulio DB, Relman DA, Brown PO: Development of the human infant intestinal microbiota. Plos Biol 2007, 5:1556-1573.

35. Koenig JE, Spor A, Scalfone N, Fricker AD, Stombaugh J, Knight R, Angenent $L T$, Ley RE: Colloquium paper: succession of microbial consortia in the developing infant gut microbiome. Proc Natl Acad Sci 2010, 108:4578-4585.

36. Biasucci G, Benenati B, Morelli L, Bessi E, Boehm G: Cesarean delivery may affect the early biodiversity of intestinal bacteria. J Nutr 2008, 138:1796S-1800S.

37. Dominguez-Bello MG, Costello EK, Contreras M, Magris M, Hidalgo G, Fiere $N$, Knight R: Delivery mode shapes the acquisition and structure of the initial microbiota across multiple body habitats in newborns. Proc Natl Acad Sci 2010, 107:11971-11975.

38. García-López R, Pérez-Brocal V, Diez-Domingo J, Moya A: Gut microbiota in children vaccinated with rotavirus vaccine. Pediatr Infect Dis J 2012, 31:1300-1302.

doi:10.1186/s13099-014-0039-8

Cite this article as: Zhang et al:: Probiotics and virulent human rotavirus modulate the transplanted human gut microbiota in gnotobiotic pigs. Gut Pathogens 2014 6:39.

\section{Submit your next manuscript to BioMed Central and take full advantage of:}

- Convenient online submission

- Thorough peer review

- No space constraints or color figure charges

- Immediate publication on acceptance

- Inclusion in PubMed, CAS, Scopus and Google Scholar

- Research which is freely available for redistribution 